\title{
Humanoid and Human Inertia Parameter Identification Using Hierarchical Optimization
}

\author{
Jovana Jovic, Adrien Escande, Ko Ayusawa, Eiichi Yoshida, Abderrahmane Kheddar, and Gentiane Venture
}

\begin{abstract}
We propose a method for estimation of humanoid and human links' inertial parameters. Our approach formulates the problem as a hierarchical quadratic program by exploiting the linear properties of rigid body dynamics with respect to the inertia parameters. In order to assess our algorithm, we conducted experiments with a humanoid robot and a human subject. We compared ground reaction forces and moments estimated from force measurements with those computed using identified inertia parameters and movement information. Our method is able to accurately reconstruct ground reaction forces and force moments. Moreover, our method is able to estimate correctly masses of the robots links and to accurately detect additional masses placed on the human subject during the experiments.
\end{abstract}

\section{INTRODUCTION}

Recently, we have witnessed considerable success with model-based preview controllers coupled with whole-body dynamic model-based controllers in achieving whole-body complex behaviors that are formulated as optimization problems in the task space (see, e.g., [1]). Being model based, the performance of such controllers depends on the relative exactness of the dynamic model, which relies in part on the body segment inertial parameters (BSIPs). Standard BSIPs of interest are mass, center of mass (CoM), location, and moment of inertia of body segments. Usually, BSIPs of a humanoid robot are provided by the manufacturer and computed using CAD software. However, those values might be inaccurate due to several reasons. Indeed, gear ratio and motor inertia can offset the ground values of the inertia parameters [2]. CAD data might include neither mass parameters of wiring and possibly light segment electronics [3] nor items that are results of hardware evolution. For example, we changed several implements in our humanoid robot, since it has been acquired: embedded PC, cameras, feet soles, and grippers have been replaced. As a consequence of changes we made during the past few years, the weight of our current humanoid robot has increased by about $5 \mathrm{~kg}$, and precise distribution of mass among segment links cannot be precisely known. Finally,

Manuscript received February 13, 2015; revised October 16, 2015; accepted April 12, 2016. Date of publication June 3, 2016; date of current version June 3, 2016. This paper was recommended for publication by Associate Editor A. Ames and Editor T. Murphey upon evaluation of the reviewers' comments.

J. Jovic, A. Escande, K. Ayusawa, and E. Yoshida are with the CNRS-AIST Joint Robotics Laboratory, UMI3218/CRT, Tsukuba 305-8568, Japan (e-mail: jovic.jovan.jovana@gmail.com; adrien_escande@yahoo.fr; k.ayusawa@aist.go.jp; e.yoshida@aist.go.jp).

A. Kheddar is with the CNRS-AIST Joint Robotics Laboratory, UMI3218/CRT, Tsukuba 305-8568 Japan, and also with the CNRS-UM2 LIRMM, UMR 5506, Interactive Digital Human Group 34095 Montpellier, France (e-mail: kheddar@gmail.com).

G. Venture is with the Department of Mechanical Systems Engineering, Tokyo University of Agriculture and Technology, Tokyo 183-0057, Japan (e-mail: venture@cc.tuat.ac.jp).

Color versions of one or more of the figures in this paper are available online at http://ieeexplore.ieee.org.

Digital Object Identifier 10.1109/TRO.2016.2558190 many laboratories have their own prototype of humanoid robot and often may not have any reliable CAD data.

In many ergonomical, biomechanical, and biomedical applications, the dynamic analysis of human subjects movement is demanded, which requires accurate estimation of standard set of BSIPs. Their misinterpretation can lead to significant variation in estimated joint kinematics [4]. In the field of biomechanics, Vaughan, Hay, and Andrews developed a method based on the optimization technique to identify inertia parameters of a human [5]. Identification of the inertia parameters consists in obtaining the dynamic model of a robot or a human that is linear with respect to the inertia parameters to be identified. Similar methods based on the optimization technique have been developed in robotics to identify BSIPs of robots with a simpler kinematic structure, such as manipulator and industrial robots [6]-[14]. Inspired by those studies, Venture et al. developed a method for identification of humanoid and human base and standard BSIPs [15]-[21]. However, we found that in some cases, the BSIPs obtained using previously cited methods could have physically inconsistent values; for example, estimated segment masses could have negative values or an inertia matrix might not be positively definite.

To overcome this drawback, we present a new formulation for the identification of BSIPs of humanoid robots and human subjects. Similarly to the work done in the field [20], [21], our method uses kinematic and ground reaction force (GRF) data obtained during the experiment to estimate BSIPs. The novelty of our method lies in using the hierarchical quadratic programming (HQP) [22] optimization technique to enforce physical consistency in the identification problem formulation. Given several groups of equality and inequality constraints and a prior order among those groups, the HQP solver finds a solution that satisfies at best in the least-squares sense the first set of constraints, the second set without worsening the satisfaction of the first set, the third set without worsening the satisfaction of the first two sets, etc. This is done by using a matrix inversion scheme based on a tailored matrix decomposition and adapting the active set approach found in quadratic programming to the hierarchical case.

The remainder of this paper has been organized as follows. Section II gives the overview of the methods developed for BSIP estimation for both humanoid robots and human subjects. Section III explains our BSIP identification problem formulation. Experiments with the HRP-2 humanoid robot and a human subject are described in Sections IV and V, respectively. Experimental data processing and analysis are detailed in Section VI. Results of BSIP identification of a humanoid robot are presented in Section VII, and those of a human in Section VIII, followed by concluding remarks and perspectives for future work. 


\section{RELATED WORK}

Several approaches have been proposed in the literature to identify the BSIPs of industrial and manipulator robots. Those methods use a dynamic model of a robot that is linear with respect to the parameters to be identified, and they solve the system using linear regression techniques such as least-squares optimization techniques [6]-[11]. In order to find physically feasible solutions, some of the methods for the BSIP identification of robots with a simple kinematic structure add numerical constraints to the optimization problem in order to force solutions to have meaningful identified BSIPs; in other words, inertia matrices of robot segments to be positive definite, masses of segments to have positive values, and, in some cases, CoM of robot segments to be inside segments volume [2], [12]. In [13][18], a knowledge of BSIP values in the form of CAD data is used to force the physical consistency of the optimization solution.

The early work in the field of human BSIP calculation was done by Dempster [25]. Dempster and his colleagues collected measurements from a few male Caucasian cadavers to create anthropometric databases that enable the segment mass to be computed from the total body mass and CoM and moment of inertia to be computed from segment length [25]-[28]. However, fluid and tissue loss in segmentation and different properties of living and deceased tissue can affect the accuracy of the estimated BSIP information, as shown in [29]. Zatsiorsky and Seluyanov determined the BSIPs from young Caucasian male and female subjects by using gamma-ray scanning technology [30]-[32]. Similar work has been performed using different medical imaging technologies, such as computed tomography [33], [34], imaging magnetic resonance [35]-[37], or X-ray technology [38]. A few authors published studies, which provide scaling equations for calculation of 3-D CoM location and 3-D moment of inertia of human body segments, based on data collected on adult living Caucasian male and female subjects [39]-[43]. Recently, the Digital Human Technology Consortium published online source of a few anthropometric databases, which provide the scaling equations for Asian population [44]. Extrapolating those data to different populations or different age subjects is restrictive due to their different body morphologies or age-related changes in anthropometry [45]. Furthermore, dynamic analysis of specific category of subjects, such as athletes or subjects with muscle atrophy due to complete spinal cord injury, requires accounting for changes in body mass distribution, which cannot be achieved using described techniques.

Subject-specific measurement of BSIPs on living humans is possible using medical imaging technologies [30]-[38]. However, those techniques are not widely used due to cost of the method, labor time during data processing, limited accessibility, and exposure of subjects to radiation in the case of imaging techniques based on emission of gamma and $\mathrm{X}$ rays.

Vaughan, Hay, and Andrews proposed a method for human BSIP identification [5]. However, the study was designed for 2-D motions in sagittal plane only. Consequently, the estimated inertia parameters are mass of segment, 2-D CoM position, and component of moment of inertia in the direction of the main axis of the segment. In addition, the formulated optimization problem was nonlinear, which results in longer computation time. Moreover, the solutions could be in local minima of the optimization function, and therefore, the results might be physically unfeasible. Recently, Venture, Ayusawa, and Nakamura proposed methods for humanoid and human base inertia parameter identification [15], [16]. The base BSIP set of human and humanoid structures is defined as a minimum set of inertia parameters that can determine the dynamic model uniquely, and they represent linear combinations of standard BSIPs [18], [19]. Contrary to the standard set of BSIPs, base BSIPs do not have a physiological meaning, and they are complicated to interpret. The method proposed in [15] and [16] has been extended to calculate standard BSIPs of humanoid robots [3], [21] and human subjects [20]. Techniques used in those studies are based on the least-squares optimization approach [3], [21]. In [20], the standard set of BSIPs was computed from the set of base BSIPs using the null space properties of a regressor matrix. The vector projected in the null space of the regressor matrix was chosen to be a set of standard parameters obtained using scaling equations from anthropometric databases. However, in some cases, solutions found with the described methods might have infeasible physical meaning.

\section{METHOD}

\section{A. Identification of Human Body Inertia Parameters}

Throughout this paper, we use the following conventions: Scalars are given in lowercase letters, matrices are given in capital letters, and vectors are given in lowercase bold letters. Notations and terms definitions are taken from [15] and [22].

The equations of dynamics of bipedal multibody systems, which are composed of $p$ rigid body segments, can be expressed as [7]

$$
\left[\begin{array}{ll}
H_{11} & H_{12} \\
H_{21} & H_{22}
\end{array}\right]\left[\begin{array}{l}
\ddot{\mathbf{q}}_{1} \\
\ddot{\mathbf{q}}_{2}
\end{array}\right]+\left[\begin{array}{l}
\mathbf{b}_{\mathbf{1}} \\
\mathbf{b}_{\mathbf{2}}
\end{array}\right]=\left[\begin{array}{l}
0 \\
\boldsymbol{\tau}
\end{array}\right]+\sum_{k=1}^{N c}\left[\begin{array}{c}
J_{1 k}^{T} \\
J_{2 k}^{T}
\end{array}\right] \mathbf{f}_{\mathbf{k}} .
$$

As defined in [11], the upper part of the equation represents motion of the base link, and the lower part describes the motion of $p$ body segments. Accordingly:

1) $q_{1}$ represents the position and orientation of base link, and $\mathbf{q}_{2}$ is the vector of joint angles of the body segments.

2) $H_{1 j}$ and $H_{2 j}(j=1,2)$ are the inertia matrices of the base link and body segments, respectively.

3) vectors $b_{1}$ and $b_{2}$ are the bias force vectors, including centrifugal, Coriolis, and gravity forces of the base link and body segments, respectively.

4) $\tau$ is the vector of joint torques of the body segments.

5) $\mathbf{f}_{\mathrm{k}}$ is the vector of the external forces at contact $k . N_{c}$ is the number of contact points with the environment.

6) $J_{1}$ and $J_{2}$ are Jacobian matrices at contact $k$ that map external forces to the joint space of the base link and body segments, respectively. 
Equation (1) can be expressed in the linear form with respect to the set of BSIPs [11]:

$$
\left[\begin{array}{l}
Y_{1} \\
Y_{2}
\end{array}\right] \boldsymbol{\phi}=\left[\begin{array}{l}
0 \\
\boldsymbol{\tau}
\end{array}\right]+\sum_{k=1}^{N c}\left[\begin{array}{l}
J_{1 k}^{T} \\
J_{2 k}^{T}
\end{array}\right] \mathbf{f}_{\mathbf{k}}
$$

where $Y=\left[\begin{array}{ll}Y_{1}^{T} & Y_{2}^{T}\end{array}\right]^{T}$ is the regressor matrix, which is a function of $\mathbf{q}_{1}$ and $\mathbf{q}_{2}$ and their derivatives; and $\phi=$ $\left[\begin{array}{llll}\phi_{0}^{T} & \phi_{1}^{T} \ldots \phi_{p}^{T}\end{array}\right]^{T}$ is a vector of BSIPs to be estimated.

For each body segment $i$, vector $\phi_{i}$ is composed of ten parameters:

1) the mass of the $i$ th segment $m_{i}$;

2) the first moment of inertia $\left[p_{x i} p_{y i} p_{z i}\right]^{T}=m_{i} \operatorname{com}_{i}$ of the segment $i$ expressed in the joint frame. Knowing the first moment of inertia of the segment $i$, its 3-D CoM position could be calculated;

3) the components $\left[i_{i x x} i_{i y y} i_{i z z} i_{i x y} i_{i x z} i_{i y z}\right]^{T}$ of the inertia matrix $I_{i}$ expressed in the joint frame,

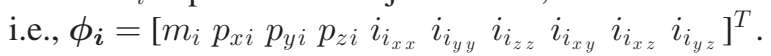

From (2), the following equation of the motion of the base link can be written:

$$
Y_{1} \phi=\sum_{k=1}^{N c} J_{1 k}^{T} \mathbf{f}_{\mathbf{k}}
$$

Taking this equation at sample times $t_{1}, \ldots, t_{n}$ along a motion data of a humanoid robot or a human subject, we obtain the following equation:

$$
\left[\begin{array}{c}
Y_{1}\left(t_{1}\right) \\
\vdots \\
Y_{1}\left(t_{n}\right)
\end{array}\right] \boldsymbol{\phi}=\sum_{k=1}^{N c}\left[\begin{array}{c}
J_{1 k}^{T} \mathbf{f}_{\mathbf{k}}\left(t_{1}\right) \\
\vdots \\
J_{1 k}^{T} \mathbf{f}_{\mathbf{k}}\left(t_{n}\right)
\end{array}\right]
$$

which we write $Y \phi=\mathbf{f}$ for the sake of clarity. Its minimumnorm least-squares solution can be computed, as in [3] and [21], by

$$
\phi=Y^{+} \mathbf{f}
$$

where $Y^{+}$is the Moore-Penrose pseudoinverse of matrix $Y$.

Using a lexicographic notation (see [22]), the above solution can be written as the solution of a two-level optimization problem, i.e.,

$$
(Y \boldsymbol{\phi}=\mathbf{f}) \succ(\phi=0) .
$$

Equation (6) means that $Y \phi=\mathbf{f}$ is solved first in the leastsquares sense, i.e., $\|Y \phi-\mathbf{f}\|^{2}$ is minimized. Then, among all possible solutions, the one that minimizes $\|\phi\|^{2}$ is chosen, solving at best $\phi=0$.

We found that due to the measurement noise and modeling errors, the solution given by (6) might not be consistent, e.g., the estimated masses of the segments can be negative, or inertial matrix may not be positive definite. Therefore, constraints are added to (5) to enforce the physical consistency of the BSIPs. We do so by formulating a four-level optimization problem that we solve with an HQP solver:

$$
\begin{aligned}
\left(\mathbf{l}_{1} \leq A_{1} \phi\right. & \left.\leq \mathbf{u}_{1}\right) \succ\left(\mathbf{l}_{2} \leq A_{2} \phi \leq \mathbf{u}_{2}\right) \\
& \succ(Y \boldsymbol{\phi}=\mathbf{f}) \succ\left(\boldsymbol{\phi}=\phi^{\mathrm{ref}}\right) .
\end{aligned}
$$

The choice of the first-level and the second-level inequality constraints is discussed below. The vector $\phi^{\text {ref }}$ contains BSIP parameters that are either those found in Dumas anthropometric databases [39], [40] when the experiment concerns human estimation, or those obtained using CAD software in the case of estimating humanoid robot BSIPs.

The first level of inequality constraints enforces physical feasibility or coherency of the estimation results. Specifically, we chose the following.

1) The mass $m_{i}$ of each body segment $i$ must be nonnegative, and the sum of all body segments masses should be equal to the total mass of the body, i.e., $m$

$$
\begin{aligned}
0 & \leq m_{i} \\
\sum_{i=1}^{p} m_{i} & =m .
\end{aligned}
$$

2) The CoM position should be inside a given convex hull of the segment volume. Since it is difficult to determine the precise geometry of human segments, and to keep linear constraints, we use a segment-axis aligned bounding box (SABB) to oversimplify both human and humanoid segment link convex hulls, i.e.

$$
\begin{aligned}
& \operatorname{com}_{x i}^{-} \leq \operatorname{com}_{x i} \leq \operatorname{com}_{x i}^{+} \\
& \operatorname{com}_{y i}^{-} \leq \operatorname{com}_{y i} \leq \operatorname{com}_{y i}^{+} \\
& \operatorname{com}_{z i}^{-} \leq \operatorname{com}_{z i} \leq \operatorname{com}_{z i}^{+}
\end{aligned}
$$

where $\operatorname{com}_{j i}$ for $j=x, y, z$ represents the 3-D CoM position of the given segment, and $\operatorname{com}_{j i}^{-}$and $\operatorname{com}_{j i}^{+}(j=x, y, z)$ define the lower and upper boundaries of the SABB, respectively. With respect to the problem variables, these constraints are rewritten as

$$
\begin{array}{ll}
0 \leq p_{x i}-m_{i} \operatorname{com}_{x i}^{-}, & p_{x i}-m_{i} \operatorname{com}_{x i}^{+} \leq 0 \\
0 \leq p_{y i}-m_{i} \operatorname{com}_{y i}^{-}, & p_{y i}-m_{i} \operatorname{com}_{y i}^{+} \leq 0 \\
0 \leq p_{z i}-m_{i} \operatorname{com}_{z i}^{-}, & p_{z i}-m_{i} \operatorname{com}_{z i}^{+} \leq 0 .
\end{array}
$$

3) The inertia matrix of each body segment $i$ must be positive definite, i.e., for every nonzero vector $\mathbf{v}$ in $\mathbb{R}^{3}$ :

$$
\mathbf{v}^{T} I_{i} \mathbf{v}>0
$$

This defines an infinite set of linear constraints, which cannot be handled by the HQP solver. We approximate it as

$$
\mathbf{v}_{\mathbf{j}}^{T} I_{i} \mathbf{v}_{\mathbf{j}} \geq \epsilon
$$

for a set of vectors $\mathbf{v}_{\mathbf{j}}$ uniformly distributed over the unit sphere and $\epsilon$ a small positive constant. By taking enough vectors and choosing a not too small $\epsilon$, this is a conservative approximation of the original constraint set. 
For each vector $\mathbf{v}_{\mathbf{j}}$ and body $i$, we get a linear inequality in the problem variables:

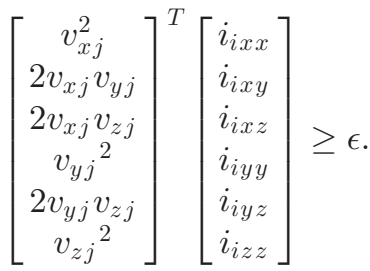

Matrix $A_{1}$ and vectors $\mathbf{l}_{\mathbf{1}}$ and $\mathbf{u}_{\mathbf{1}}$ are readily built from (8)(14), with components of $\mathbf{l}_{1}$ and $\mathbf{u}_{1}$ being equal for an equality constraint and set to $-\infty$ (respectively, $+\infty$ ) when the lower bound (respectively, upper bound) of an inequality constraint is not specified.

The second level of optimization inequality constraints is chosen to:

1) enforce symmetry between the BSIPs of the left side of the body and the BSIPs of the right side of the body $i$, when this is the case, by applying the following constraints:

$$
\begin{aligned}
& (1-\alpha) \mathbf{x}_{\mathbf{i}} \leq \mathbf{x}_{\mathbf{i}} \leq(1+\alpha) \mathbf{x}_{\mathbf{i}} \\
& (1-\alpha) \mathbf{x}_{\mathbf{j}} \leq \mathbf{x}_{\mathbf{j}} \leq(1+\alpha) \mathbf{x}_{\mathbf{j}}
\end{aligned}
$$

where $\mathbf{x}$ is a vector of body segment masses and diagonal elements of inertia matrix, and $\alpha$ is a constant chosen so that $0<\alpha<1$.

2) For the mass of each segment $i$, we imposed the following:

$$
(1-\beta) m_{i}^{\text {ref }} \leq m_{i} \leq(1+\beta) m_{i}^{\text {ref }}
$$

where $m_{i}^{\text {ref }}$ is the value of mass of the segment $i$ taken form $\phi^{\text {ref }}$, and $0<\beta<1$.

$A_{2}, \mathbf{l}_{2}$, and $\mathbf{u}_{2}$ are easily built from (15) and (16).

The constraints of the second level are applied only in the case of healthy subjects. Otherwise, such as in stroke survival cases, those inequality constraints should be modified to include left- and right-side mass asymmetry if needed.

\section{B. Numerical Values}

In this study, we chose 10242 vectors $\mathbf{v}_{\mathbf{j}}$ corresponding to subdividing iteratively five times the faces of a regular icosahedron. ${ }^{1}$ Constants $\epsilon$ and $\alpha$ have values 0.01 and 0.1 , respectively. The choice of constant $\beta$ is discussed in Sections VII and VIII.

\section{Humanoid CASE STUdy: IDENTIFYING HRP-2 INERTIA PARAMETERS}

We use our HRP-2 robot, which is presented in Fig. 1, for the case study. The robot is $1.54 \mathrm{~m}$ high, with the CAD mass of $58 \mathrm{~kg}$. It is composed of 31 segment links and has 30 degrees of freedom (DoFs), not counting the free flyer: six at each leg, seven at each arm, two at the waist, and two at the neck. The robot is equipped with sensors measuring the joint angles, contact reaction forces under the feet, accelerometer, and gyroscope in the trunk segment [46].

\footnotetext{
${ }^{1}$ This is a classical way to obtain evenly distributed points on a sphere.
}
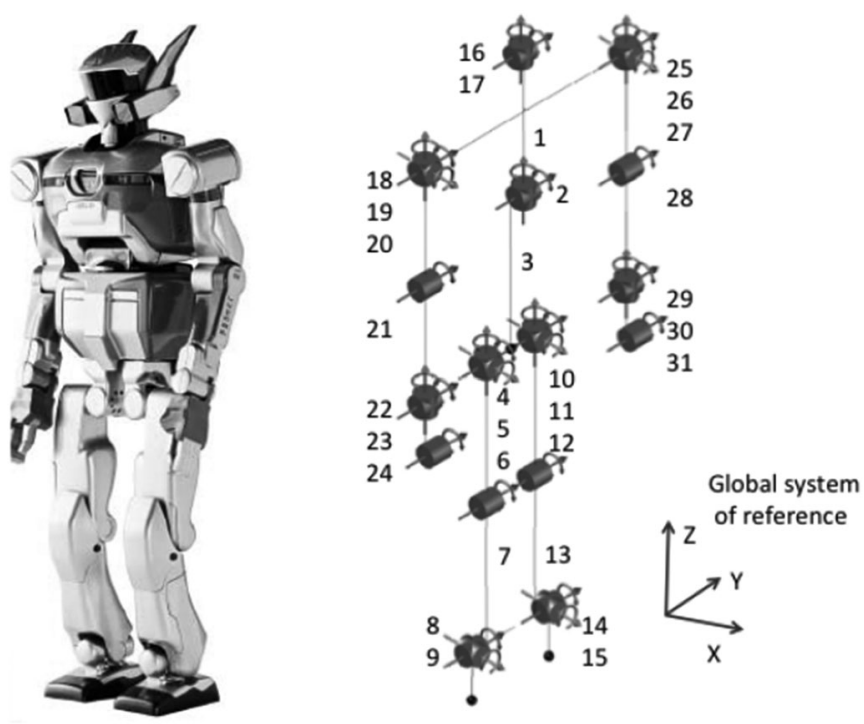

Fig. 1. HRP-2 humanoid robot and its kinematics tree.

Since its acquisition, several hardware changes were progressively made on the HRP-2 (some parts were added or replaced: embedded PC located in trunk segments, camera sensors located in the head segment, feet soles located in feet, as well as force sensors located in the hand segments were changed, and one DoF in each wrist was added). These modifications resulted in an increase of the total mass of the robot by $5 \mathrm{~kg}$, which involved de facto changes of its segment inertial properties. Consequently, BSIP information obtained by CAD software could have an estimation error up to $50 \%$.

We conducted experiments with the HRP-2 humanoid robot in order to build a database of humanoid joint angles trajectories and GRFs to feed the BSIP identification process. The accuracy of the identified BSIPs depends on the richness of the motion. Such motions are often called exciting trajectories, and they are defined as whole-body movements that provide sufficient kinematic information for accurate segment mass parameter estimation [11]. In this case study, the method for generating exciting trajectories of a robot is inspired by the method designed for choosing the motion with optimal excitation properties for identification of human inertia parameters [16]. The method generates random joint trajectories for each DoF of the humanoid robot, taking into account dynamic stability of the humanoid robotic structure and ensuring self-collision free motions. Using generated joint trajectories, it creates the regressor matrix and decomposes it into elementary subregressors. It further computes condition numbers for each of these subregressors that represent the precision of the identified mass parameters [16]. Finally, for each DoF of the humanoid robot, it chooses joint trajectories with the lower value of the condition number [17]. The exciting trajectories were executed by our HRP-2 robot. The base link is chosen to be at the trunk segment, which contains both the accelerometer and gyroscope sensors. Examples of the performed motions are shown in Fig. 2. Outputs of sensors of HRP-2 robot were recorded with 200-Hz sampling frequency. 


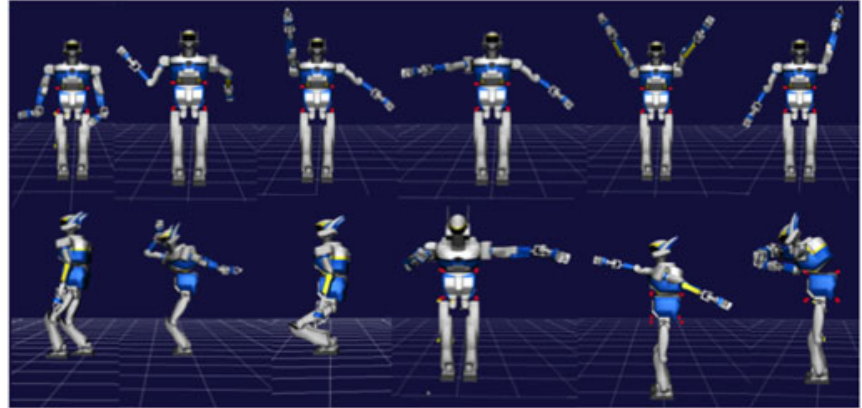

Fig. 2. Examples of exciting trajectories executed by the HRP-2 humanoid robot.

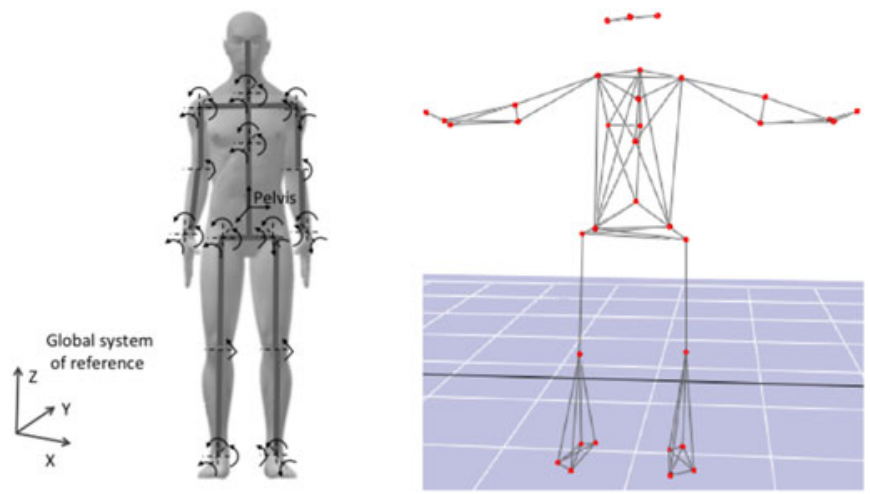

Fig. 3. (a) Biomechanical model of the human body. (b) Reflective marker positions (in red) and initial position of the subject.

\section{Human CASE STUdy}

The biomechanical model of a human body shown in Fig. 3 is used in this case study. The model consists of 15 segments, ten spherical joints and four revolute joints, i.e., 34 DoF. Segment frames are defined at the joint centers of rotation. We assume that spherical and revolute joints could describe the behavior of the whole-body motion quite well.

One healthy adult (male, $70 \mathrm{~kg}, 1.82 \mathrm{~m}$ ) volunteered in the study. The experimental procedure is in accordance with the Helsinki Declaration of 2000. The choice of the identification motion of the human subject is based on literature review [16]. Starting from the initial position shown in Fig. 3, the participant performed a motion that excites the whole-body dynamics, as defined in [16]. Examples of the performed motions are shown in Fig. 4. In order to test the ability of the algorithm in detecting segment mass changes, the participant was asked to perform three validation trials executing the same motion and wearing a weighted belt located at the lower trunk level in the first validation trial, at the right hand in the second validation trial, and at the left foot during the third validation trial. The mass of the belt was $2 \mathrm{~kg}$.

Kinematic variables were measured using a stereophotogrammetric system (motion analysis) with $10-\mathrm{Mx}$ cameras. The sampling frequency of cameras was $200 \mathrm{~Hz}$. In order to accurately estimate small postural modifications, 35 reflective markers were located over the left and right head temple; over the parietal bone; over the seventh cervical vertebrae; over the tenth

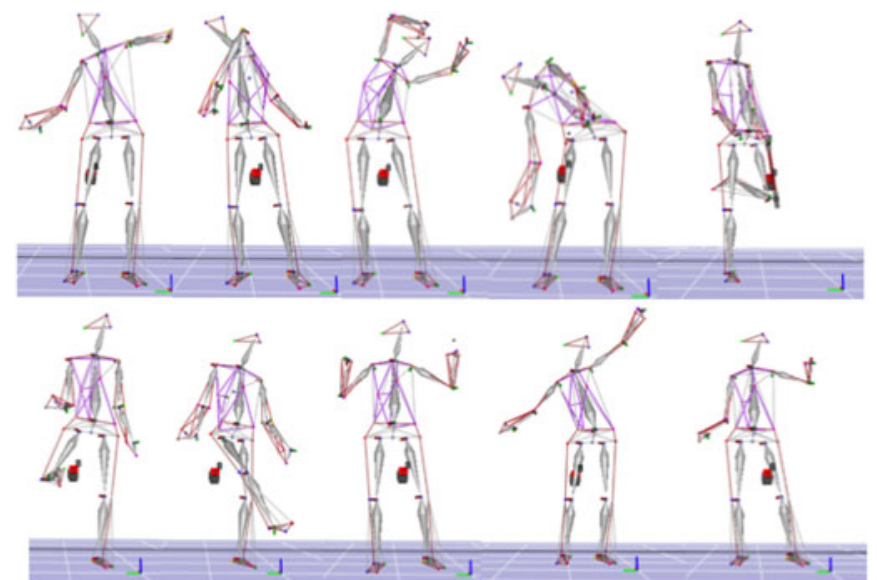

Fig. 4. Examples of identification motions visualized in Cortex motion analysis software.

thoracic vertebrae; in the middle of the right scapula; over the Suprasternal notch; over the Xiphoid process, on the mid-way between the posterior superior iliac spines; on both legs: on the heels, the first and the fifth metatarsal heads, the lateral malleoluses, on the lateral condyles, over the great trochanters, the anterior superior iliac spines; and on both arms: on the acromio-clavicular joints, on the lateral and medial epicondyles, on the wrist bars at the thumb and pinkie sides, and at the heads of the second metacarpal. The positions of reflective markers are presented in Fig. 3. GRF was simultaneously measured using two force plates (Kistler) with a sampling frequency of $1000 \mathrm{~Hz}$.

\section{DATA ANALYSIS}

The outputs of the HRP-2 sensors are filtered using a thirdorder low-pass Butterworth filter with the cutoff frequency of $20 \mathrm{~Hz}$. The joint angles, associated with the dynamical model of a human defined in the previous section, are estimated from the marker positions using Cortex Motion Analysis software. The 3-D marker positions were filtered using a third-order lowpass Butterworth filter implemented in Cortex Motion Analysis software [47] with the cutoff frequency of $6 \mathrm{~Hz}$ in accordance with investigations done in [48] and [49]. The estimation of BSIPs of humanoid and human models is done using in-house software that executes the algorithm described in Section III.

In both BSIP identification cases, the root-mean-square error (RMSE) and Pearson's correlation coefficient (CC) are computed between the GRFs and respective ground reaction force moments (GRFMs) reconstructed from motion data and identified BSIPs and those obtained from the force plates measurements. The results are compared with the RMSE and Pearson's $\mathrm{CC}$ values computed between GRFs and GRFMs estimated from the force plate data and those computed using motion data and BSIPs given by CAD software in the case of humanoid BSIPs identification, and found in Dumas anthropometric database in the case of human BSIP identification [39], [40]. 
TABLE I

RMSE AND PEARSON'S CC VALUES BETWEEN THE GRF AND GRFM MEASURED Using Force SENSORS AND THOSE ReCONSTRUCTED Using IDENTIFIED AND CAD DATA FOR HRP-2 HUMANOID ROBOT

\begin{tabular}{llccccc}
\hline \hline & \multicolumn{2}{c}{ GRF } & & \multicolumn{2}{c}{ GRFM } \\
\cline { 3 - 4 } \cline { 5 - 6 } \cline { 5 - 6 } & & Identified BSIPs & CAD BSIPs & Identified BSIPs & CAD BSIPs \\
\hline$Z$-direction & RMSE & $37.20[\mathrm{~N}]$ & $52.50[\mathrm{~N}]$ & & $1.70[\mathrm{~N} \cdot \mathrm{m}]$ & $2.10[\mathrm{~N} \cdot \mathrm{m}]$ \\
& Pearson's CC & 0.65 & 0.63 & & 0.75 & 0.69 \\
$Y$-direction & RMSE & $13.40[\mathrm{~N}]$ & $17.90[\mathrm{~N}]$ & & $5.00[\mathrm{~N} \cdot \mathrm{m}]$ & $9.40[\mathrm{~N} \cdot \mathrm{m}]$ \\
& Pearson's CC & 0.88 & 0.77 & & 0.95 & 0.87 \\
$X$-direction & RMSE & $14.60[\mathrm{~N}]$ & $15.80[\mathrm{~N}]$ & & $7.00[\mathrm{~N} \cdot \mathrm{m}]$ & $11.40[\mathrm{~N} \cdot \mathrm{m}]$ \\
& Pearson's CC & 0.75 & 0.65 & 0.85 & 0.71 \\
\hline \hline
\end{tabular}
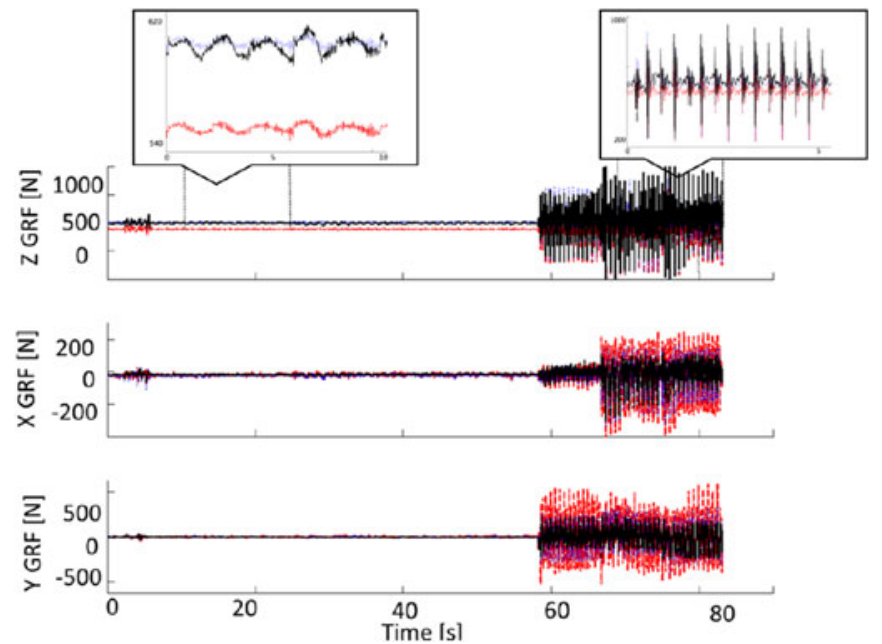

Fig. 5. GRFs in vertical and horizontal directions reconstructed using identified BSIPs (blue dotted line) and CAD BSIPs (red dashed line), obtained using force sensors measurements (black solid line).

\section{Results OF HRP-2 HumanoID Robot Body SEGMENT INERTIAL PARAMETER IDENTIFICATION}

Since no ground-truth values of BSIPs can be known in practice, we assessed our method by comparing GRFs and moments, which are reconstructed using identified BSIPs, with force measurements counterparts. The results are given in Table I. Figs. 5 and 6 show reconstructed GRFs and moments reconstructed from identified inertia parameters and from CAD data with respect to measured ones. Our method provides accurate reconstruction of GRFs and moments relatively to those reconstructed using CAD data. The mass of each segment link obtained using our method and the ones given by CAD software is presented in Fig. 7 and Table II. ${ }^{2}$ The difference between the identified and CAD mass values of unmodified segment 5 and unmodified segment 11 is probably because of poor excitation of those segments that we could not improve due to stability reasons.

The choice of the value of the second-level optimization constant was based on the knowledge of the replaced components in the modified robot segments. For modified robot segments,

\footnotetext{
${ }^{2}$ Identified segments mass values are expressed in percentage of CAD segment mass values due to confidentiality issues.
}

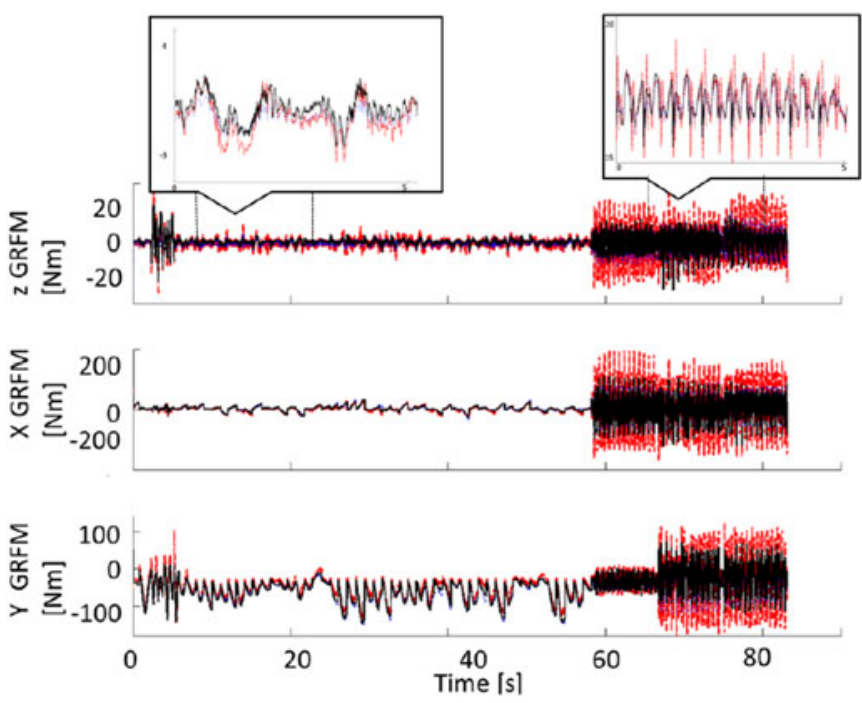

Fig. 6. GRFMs in vertical and horizontal directions reconstructed using identified BSIPs (blue dotted line) and CAD BSIPs (red dashed line), obtained using force sensors measurements (black solid line).

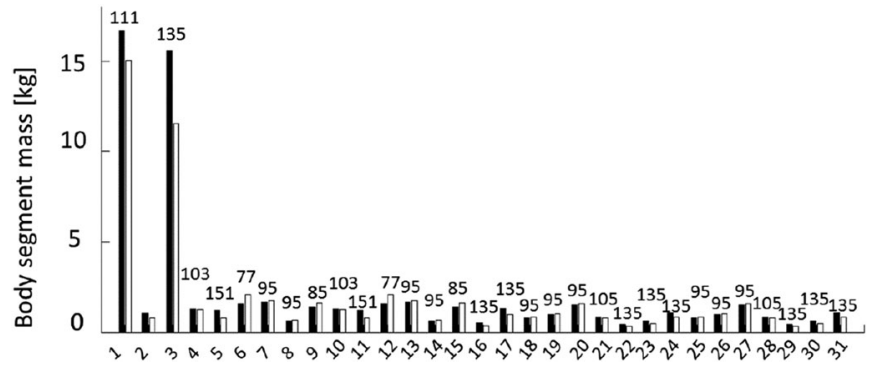

Fig. 7. Identified body segment masses (white) and body segment masses extracted from CAD data (black). Identified BSIPs are expressed as percentage of CAD BSIPs above corresponding bars. For segment link location information, see Fig. 1.

$\beta$ is 0.35 , and 0.05 for segments whose inertia parameters are expected to be close to CAD data.

\section{Results OF Human Body SEgment INERTIAL PARAMETER IDENTIFICATION}

Table III presents the comparison between GRFs and moments reconstructed using identified BSIPs and BSIPs found in the Dumas database with those obtained using the force plate measurements. GRFs and GRFMs reconstructed using our method (blue dotted line), Dumas database (red dashed line), and those obtained using the force plates measurement (black line) are shown in Figs. 8 and 9, respectively. We can see that our method is able to reconstruct GRFs and GRFMs more accurately compared with the method based on the use of anthropometric databases.

The subject's body segment masses obtained using described method and the ones calculated using scaling equations found in the Dumas database are presented in Fig. 10. In order to test the ability of the proposed method to detect body segment mass changes, the participant was asked to perform three validation trials wearing a $2-\mathrm{kg}$ weighted belt. The identified segment 
TABLE II

Identified Body Segment Masses [\% of CAD Segment Masses]

\begin{tabular}{|c|c|c|c|c|c|c|c|}
\hline \multicolumn{5}{|c|}{ Torso and Head } & \multicolumn{3}{|c|}{ Right Leg } \\
\hline $\begin{array}{l}\text { Segment } 1 \\
111.03\end{array}$ & $\begin{array}{c}\text { Segment } 2 \\
135.00\end{array}$ & $\begin{array}{c}\text { Segment } 3 \\
135.00\end{array}$ & $\begin{array}{c}\text { Segment } 16 \\
135.00\end{array}$ & $\begin{array}{c}\text { Segment } 17 \\
135.00\end{array}$ & $\begin{array}{c}\text { Segment } 4 \\
102.94\end{array}$ & $\begin{array}{c}\text { Segment } 5 \\
151.23\end{array}$ & $\begin{array}{c}\text { Segment } 6 \\
77.42\end{array}$ \\
\hline \multicolumn{3}{|c|}{ Right Leg } & \multicolumn{5}{|c|}{ Left Leg } \\
\hline $\begin{array}{l}\text { Segment } 7 \\
95.00\end{array}$ & $\begin{array}{l}\text { Segment } 8 \\
95.00\end{array}$ & $\begin{array}{c}\text { Segment } 9 \\
84.99\end{array}$ & $\begin{array}{c}\text { Segment } 10 \\
102.94\end{array}$ & $\begin{array}{c}\text { Segment } 11 \\
151.23\end{array}$ & $\begin{array}{c}\text { Segment } 12 \\
77.42\end{array}$ & $\begin{array}{c}\text { Segment } 13 \\
95.00\end{array}$ & $\begin{array}{c}\text { Segment } 14 \\
95.00\end{array}$ \\
\hline Left Leg & \multicolumn{7}{|c|}{ Right Arm } \\
\hline $\begin{array}{l}\text { Segment } 15 \\
84.99\end{array}$ & $\begin{array}{c}\text { Segment } 18 \\
95.00\end{array}$ & $\begin{array}{c}\text { Segment } 19 \\
95.00\end{array}$ & $\begin{array}{c}\text { Segment } 20 \\
95.00\end{array}$ & $\begin{array}{c}\text { Segment } 21 \\
104.99\end{array}$ & $\begin{array}{c}\text { Segment } 22 \\
135.00\end{array}$ & $\begin{array}{c}\text { Segment } 23 \\
134.99\end{array}$ & $\begin{array}{c}\text { Segment } 24 \\
135.00\end{array}$ \\
\hline \multicolumn{8}{|c|}{ Left Arm } \\
\hline $\begin{array}{l}\text { Segment } 25 \\
95.00\end{array}$ & $\begin{array}{c}\text { Segment } 26 \\
95.00\end{array}$ & $\begin{array}{c}\text { Segment } 27 \\
95.00\end{array}$ & $\begin{array}{c}\text { Segment } 28 \\
104.99\end{array}$ & $\begin{array}{c}\text { Segment } 29 \\
135.00\end{array}$ & $\begin{array}{c}\text { Segment } 30 \\
134.99\end{array}$ & $\begin{array}{c}\text { Segment } 31 \\
135.00\end{array}$ & \\
\hline
\end{tabular}

For segment link location information, see Fig. 1.

TABLE III

RMSE AND PEARson's CC VALUES BETWEen the GRFs AND RESPECTIVE GRFMS Estimated From the ForCE Plate MEASUREMENTS AND THOSE RECONSTRUCTED USING IDENTIFIED BSIPS AND BSIPS FOUND IN THE DUMAS DATABASE FOR A HUMAN SUBJECT

\begin{tabular}{cccccc}
\hline \hline & \multicolumn{2}{c}{ GRF } & \multicolumn{2}{c}{ GRFM } \\
\cline { 2 - 3 } \cline { 5 - 6 } & \multicolumn{2}{c}{ Identified BSIPs Dumas BSIPs Identified BSIPs Dumas BSIPs } \\
\hline$Z$-direction RMSE & $8.65[\mathrm{~N}]$ & $9.62[\mathrm{~N}]$ & & $9[\mathrm{~N} \cdot \mathrm{m}]$ & $9[\mathrm{~N} \cdot \mathrm{m}]$ \\
Pearson's CC & 0.94 & 0.92 & 0.52 & 0.40 \\
$X$-direction RMSE & $0.18[\mathrm{~N}]$ & $0.18[\mathrm{~N}]$ & $26.80[\mathrm{~N} \cdot \mathrm{m}]$ & $51.40[\mathrm{~N} \cdot \mathrm{m}]$ \\
Pearson's CC & 0.40 & 0.40 & 0.43 & -0.09 \\
$Y$-direction RMSE & $2.95[\mathrm{~N}]$ & $2.95[\mathrm{~N}]$ & $19.40[\mathrm{~N} \cdot \mathrm{m}]$ & $58[\mathrm{~N} \cdot \mathrm{m}]$ \\
Pearson's CC & 0.68 & 0.68 & 0.78 & 0.58 \\
\hline \hline
\end{tabular}
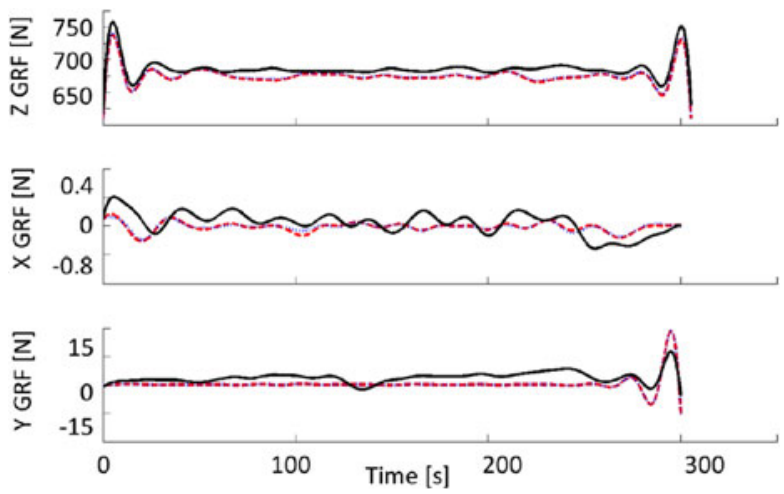

Fig. 8. GRFs in vertical and horizontal directions reconstructed using identified BSIPs (blue dotted line) and BSIPs from the Dumas database (red dashed line), obtained using force plates measurements (black solid line).

masses for all trials, together with segment masses calculated using scaling equations form database of Dumas et al., are given in Table IV and presented in Figs. 11-13. The belt weight estimations during all validation trials are given in the last row of Table IV. The commonly used methods for estimation of human BSIPs based on anthropometric databases suppose symmetry of the left and right sides of the human body. Depending on the targeted group of the subject, such as professional tennis play-
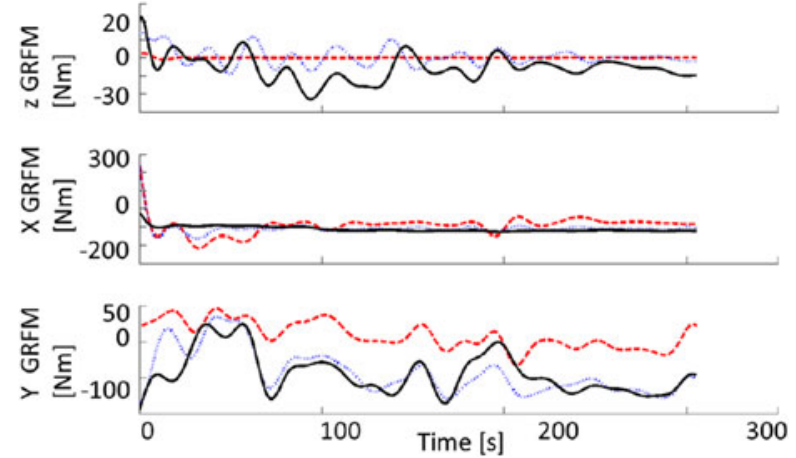

Fig. 9. GRFMs in vertical and horizontal directions reconstructed using identified BSIPs (blue dotted line) and BSIPs from the Dumas database (red dashed line line), obtained using force plates measurements (black solid line).

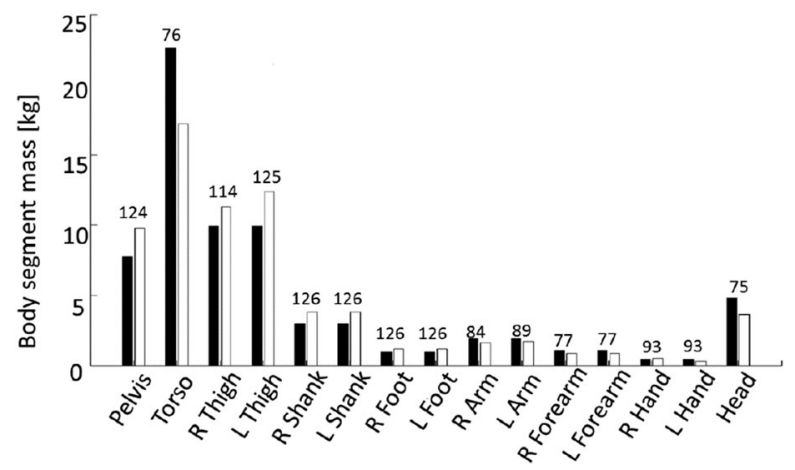

Fig. 10. Identified body segment masses (white) and body segment masses from the Dumas database (black). Right and left sides of the human body are abbreviated with $\mathrm{R}$ and L, respectively. Identified BSIPs are expressed as percentage of BSIPs from the Dumas database above corresponding bars.

ers [50] or stroke survivals [51], that might not be the case. As we can see from presented results, our method exhibits good performances in estimation of the additional segment masses, contrary to the database of Dumas et al.

For validation trials, the second-level optimization inequality constraints are modified. As the mass of the weighted belt exceeds $10 \%$ of the mass of the body segment where the belt 
TABLE IV

Identified Body Segment Masses and Body Segment Masses Found in the Dumas Database For All Performed TRIals [KG]

\begin{tabular}{|c|c|c|c|c|c|c|c|c|}
\hline & \multicolumn{2}{|c|}{ No extra weight } & \multicolumn{2}{|c|}{$2 \mathrm{~kg}$ on the lower trunk level } & \multicolumn{2}{|c|}{$2 \mathrm{~kg}$ on the right hand level } & \multicolumn{2}{|c|}{$2 \mathrm{~kg}$ on the left foot level } \\
\hline & Dumas & Identified & Dumas & Identified & Dumas & Identified & Dumas & Identified \\
\hline Pelvis & 7.80 & 9.70 & 8 & 12.20 & 8 & 10 & 8 & 10 \\
\hline Torso & 22.60 & 17.20 & 23.20 & 17.40 & 23.20 & 17.40 & 23.20 & 17.40 \\
\hline Right Thigh & 9.90 & 11.30 & 10.20 & 11.20 & 10.20 & 11.30 & 10.20 & 11 \\
\hline Left Thigh & 9.90 & 12.40 & 10.20 & 12.30 & 10.20 & 12.50 & 10.20 & 12 \\
\hline Right Shank & 3 & 3.80 & 3.10 & 3.50 & 3.10 & 3.70 & 3.10 & 3.50 \\
\hline Left Shank & 3 & 3.80 & 3.10 & 3.90 & 3.10 & 3.90 & 3.10 & 3.50 \\
\hline Right Foot & 0.95 & 1.20 & 1 & 0.90 & 1 & 1.10 & 1 & 0.90 \\
\hline Left Foot & 0.95 & 1.20 & 1 & 1 & 1 & 1 & 1 & 3.20 \\
\hline Right Arm & 1.90 & 1.60 & 1.95 & 1.60 & 1.95 & 1.60 & 1.95 & 1.60 \\
\hline Left Arm & 1.90 & 1.70 & 1.95 & 1.60 & 1.95 & 1.60 & 1.95 & 1.60 \\
\hline Right Forearm & 1.10 & 0.85 & 1.20 & 0.70 & 1.20 & 0.80 & 1.20 & 1.10 \\
\hline Left Forearm & 1.10 & 0.85 & 1.20 & 0.90 & 1.20 & 0.80 & 1.20 & 1.10 \\
\hline Right Hand & 0.43 & 0.40 & 0.44 & 0.50 & 0.44 & 1.90 & 0.44 & 0.50 \\
\hline Left Hand & 0.43 & 0.40 & 0.44 & 0.40 & 0.44 & 0.40 & 0.44 & 0.40 \\
\hline Head & 4.80 & 3.60 & 5 & 3.70 & 5 & 3.70 & 5 & 4 \\
\hline Belt weight estimation & / & l & 0.20 & 2.50 & 0.01 & 1.50 & 0.05 & 2 \\
\hline
\end{tabular}

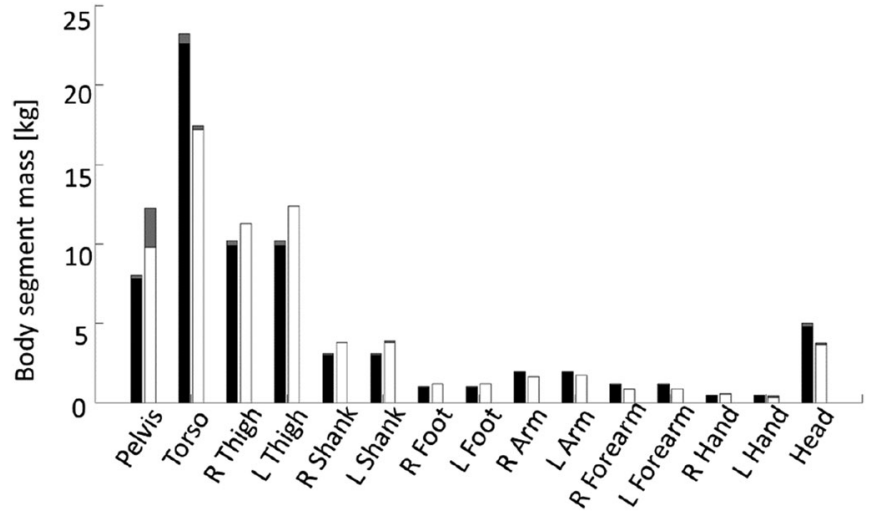

Fig. 11. Segment mass increase (gray) of identified body segment masses (white) and body segment masses from the Dumas database (black) for the validation trial subject performed wearing the weighted belt located on the lower trunk level. Right and left sides of the human body are abbreviated with $\mathrm{R}$ and $\mathrm{L}$, respectively.

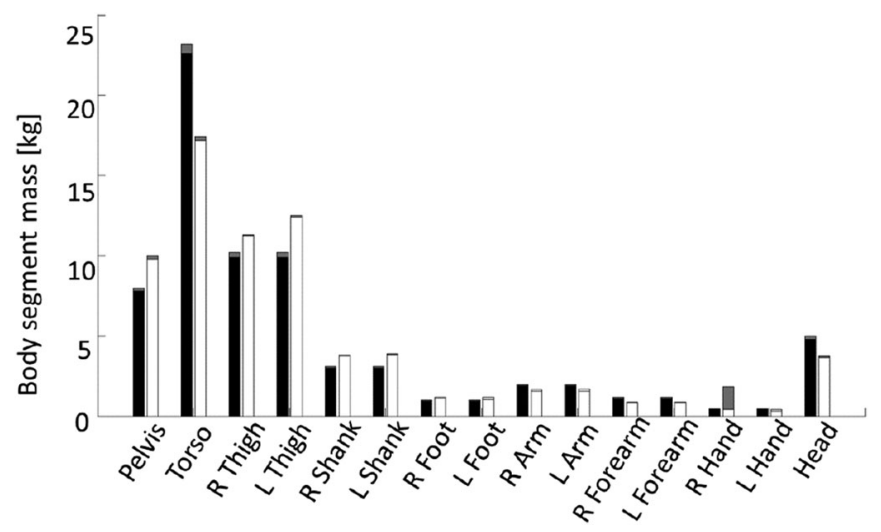

Fig. 12. Segment mass increase (gray) of identified body segment masses (white) and body segment masses from the Dumas database (black) for the validation trial subject performed wearing the weighted belt located on the right-hand level. Right and left sides of the human body are abbreviated with $\mathrm{R}$ and $\mathrm{L}$, respectively.

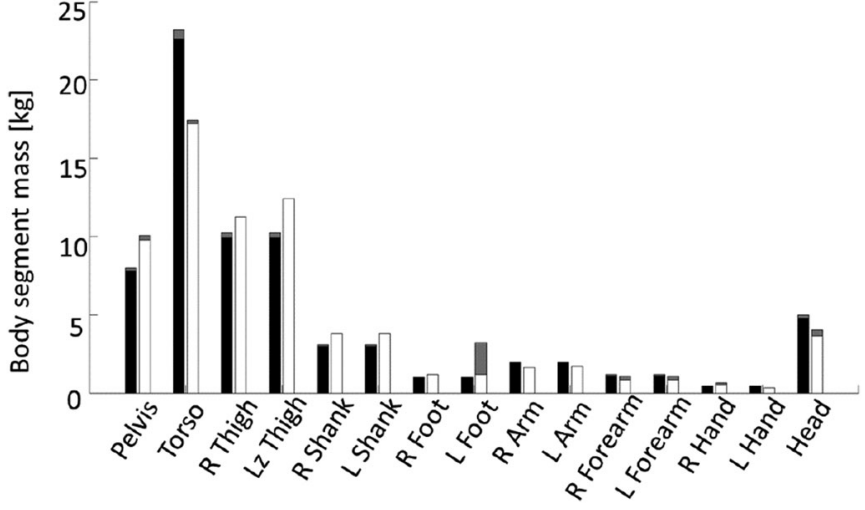

Fig. 13. Segment mass increase (gray) of identified body segment masses (white) and body segment masses from the Dumas database (black) for the validation trial subject performed wearing the weighted belt located on the leftfoot level. Right and left sides of the human body are abbreviated with R and L, respectively.

was located, the inequality constraint forcing the symmetry of the BSIPs of the left and the right sides of the human body is removed. Values of $m^{\text {ref }}$ parameters in (16) were modified for the pelvis, right hand, and left foot body segments by adding the mass of the weighted belt. The value of constant $\beta$ is 0.35 .

\section{CONCLUSION}

In this study, we have formulated the humanoid/human inertial parameter identification as an HQP optimization problem whose inputs are the kinematic and GRF and GRFM measurements.

In the humanoid BSIP case study, we compared results obtained using our method with the results obtained using available robot's segment inertia parameters, i.e., CAD software data. Our method is able to reconstruct the GRFs and moments more accurately relatively to using CAD data. In addition, we estimate correctly masses of the modified segment links of the HRP-2 robot that we believe are closer to the reality. 
In the human BSIP identification case study, we compared results obtained from our method with those obtained using the anthropometric database, which is the most common method for human BSIP identification. The anthropometric database published in [39] and [40] is chosen because it provides 3-D location of the segment CoM positions. In addition, the scaling equations are computed from anthropometric data of the living subjects. Comparing with the other studies of similar characteristics [41], [42], the Dumas anthropometric database provides the scaling equations for BSIPs expressed in the segment coordinate systems. Contrary to the latter approach, our method exhibits better performances in the estimation of the atypical antisymmetric segment weights and is able to reconstruct the GRFs and moments more accurately.

As stated earlier, we found that methods based on the leastsquares approach [see (5)] [3], [21] often might lead to physically inconsistent values. The typical example of those solutions would be negative values of some segment masses. Applying the additional constraints [see (7)] to the optimization function, we ensure physical coherency of the results.

We approximate the positive definiteness of the inertia matrix [see (12)-(14)]; however, there is no coupling among the mass of a body segment, its CoM position, and its inertia tensors. Future studies will address this problem by defining the relationship among inertia tensors, geometry, and mass distribution of the body segments, as done in [52].

\section{ACKNOWLEDGMENT}

The authors would like to thank D. Dimitrov and P.-B. Wieber for providing their implementation of the hierarchical solver and for various interesting discussions. They would also like to thank the staff of the Nakamura Laboratory, University of Tokyo, for providing them with the captured human motions.

This research is partially supported by the Japan Society for the Promotion of Science (JSPS) Grant-in-Aid for JSPS Fellows (25-03796 and 25-03797) and the New Energy and Industrial Technology Development Organization (NEDO) and the International R\&D and Demonstration Project on Robotics Field (USA) and the R\&D on Disaster Response Robot simulator based on the Choreonoid framework.

\section{REFERENCES}

[1] H. Audren, J. Vaillant, A. Kheddar, A. Escande, K. Kaneko, and E. Yoshida, "Model preview control in multi-contact motion-application to a humanoid robot," in Proc. IEEE Int. Conf. Intell. Robots Syst, Chicago, IL, USA, 2014, pp. 4030-4035.

[2] R. S. Jamisola, Jr. and E. P. Dadios, "Identifying moments of inertia parameters for rigid-body manipulators," presented at the Int. Conf. Mechatron. Technol., Cebu City, Philippines, 2009.

[3] K. Ayusawa, G. Venture, and Y. Nakamura, "Identification of the inertial parameters of a humanoid robot using unactuated dynamics of the base link," presented at the IEEE-RAS Int. Conf. Humanoid Robots, Daejeon, South Korea, 2008.

[4] G. Rao, D. Amaratini, E. Breton, and D. Favier, "Influence of body segments' parameters estimation models on inverse dynamic solutions during gait," J. Biomech. vol. 39, pp. 1531-1536, 2005.

[5] C. L. Vaughan, J. G. Hay, and J. G. Andrews, "Selection of body segment parameters by optimization methods," ASME J. Biomech. Eng., vol. 104 , no. 3, pp. 38-44, 1982.

[6] K. Kozlowski, Modelling and Identification in Robotics. London, U.K.: Springer-Verlag, 1998.
[7] K. Yamane, Simulating and Generating Motions of Human Figures (Springer Tracts in Advanced Robotics). Heidelberg, Germany: SpringerVerlag, 2004

[8] K. Ayusawa, G. Venture, and Y. Nakamura, "Identifiability and identification of inertial parameters using the underactuated base-link dynamics for legged multibody systems," Int. J. Robot. Res., vol. 33, no. 3, pp. 446-468, 2014.

[9] M. Gautier and W. Khalil, "Exciting trajectories for the identification of base inertial parameters of robots," Int. J. Robot. Res., vol. 11, no. 4, pp. 362-375, 1992.

[10] A. Kinsheel and Z. Taha, "Identification of the parameters of robot manipulators dynamics about an operating point using perturbed dynamics," presented at the 11th Int. Conf. Control, Autom., Robot. Vision, Singapore, 2010.

[11] J. Swevers, C. Ganseman, B. D. Tukel, J. De Schutter, and H. Van Brussel, "Optimal robot excitation and identification," IEEE Trans. Robot. Autom., vol. 13, no. 5, pp. 730-740, Oct. 1997.

[12] M. Diaz-Rodrigueza, V. Matab, A. Valerac, and A. Paged, "A methodology for dynamic parameters identification of 3-DOF parallel robots in terms of relevant parameters," Mech. Mach. Theory, vol. 45, no. 9, pp. 1337-1356, 2010.

[13] M. Gautier, S. Briot, and G. Venture, "Identification of consistent standard dynamic parameters of industrial robots," presented at the IEEE Int. Conf. Adv. Intell. Mechatron., Wollongong, Australia, 2013.

[14] M. Gautier and G. Venture, "Identification of standard dynamic parameters of robots with positive definite inertia matrix," presented at the IEEE Int. Conf. Intell. Robots Syst, Tokyo, Japan, 2013.

[15] G. Venture, K. Ayusawa, and Y. Nakamura, "Identification of human mass properties from motion," presented at the IFAC Int. Conf. Syst. Identification, Saint Malo, France, 2009.

[16] G. Venture, K. Ayusawa, and Y. Nakamura, "A numerical method for choosing motions with optimal excitation properties for identification of biped dynamics-An application to human," presented at the IEEE/RAS Int. Conf. Robot. Autom., Kobe, Japan, 2009.

[17] J. Jovic, F. Philipp, A. Escande, K. Ayusawa, E. Yoshida, A. Kheddar, and G. Venture, "Identification of dynamics of humanoids: Systematic exciting motion generation," in Proc. IEEE/RSJ Int. Conf. Intell. Robots Syst., 2015, pp. 2762-2767.

[18] H. Mayeda, K. Yoshida, and K. Osuka, "Base parameters of manipulator dynamic models," IEEE Trans. Robot. Autom., vol. 6, no. 3, pp. 312-321, 1990.

[19] P. Khosla, "Estimation of robot dynamics parameters: Theory and application," Carnegie Mellon University, Pittsburgh, PA, USA, Tech. Rep., 1987.

[20] G. Venture, K. Ayusawa, and Y. Nakamura, "Real-time identification and visualization of human segment parameters," presented at the IEEE/EMBC Int. Conf. Eng. Med. Biol., Minneapolis, MN, USA, 2009.

[21] T. Iwasaki, G. Venture, and E. Yoshida, "Identification of the inertial parameters of a humanoid robot using grounded sole link," presented at the IEEE-RAS Int. Conf. Humanoid Robots, Osaka, Japan, 2012.

[22] A. Escande, N. Mansard, and P. B. Wieber, "Hierarchical quadratic programming: Fast online humanoid-robot motion generation," Int. J. Robot. Res., vol. 33, no. 7, pp 1006-1028, 2014.

[23] M. De Lasa, I. Mordatch, and A. Hertzmann, "Feature-based locomotion controllers," in Proc. ACM SIGGRAPH, Los Angeles, CA, USA, 2010, p. 131.

[24] P. Kanoun, F. Lamiraux, and P. B. Wieber, "Kinematic control of redundant manipulators: Generalizing the task priority framework to inequality tasks," IEEE Trans. Robot., vol. 27, no. 4, pp. 785-792, Aug. 2011.

[25] W. T. Dempster, "Space requirements of the seated operator," Aerosp. Med. Res. Lab., Wright-Patterson Air Force Base, OH, USA, WADC Tech. Rep. 55-159, 1955.

[26] R. F. Chandler, C. E. Clauser, J. T. Mcconville, H. M. Reynolds, and J. W. Young, "Investigation of inertial properties of the human body," Aerosp. Med. Res. Lab., Wright-Patterson Air Force Base, OH, USA, WADC Tech. Rep. AMRL-TR-70-137, 1975.

[27] C. E. Clauser, J. T. Mconville, and J. W. Young, "Weight, volume, and center of mass of segments of the human body," Aerosp. Med. Res. Lab., Wright-Patterson Air Force Base, OH, USA, WADC Tech. Rep. AMRLTL-69-70, 1969.

[28] D. A. Winter, The Biomechanics and Motor Control of Human Gait: Normal, Elderly and Pathological. Waterloo, ON, Canada: Univ. Waterloo Press, 1991. 
[29] J. L. Durkin, "Measurement and estimation of human body segment parameters," in, Routledge Handbook of Biomechanics and Human Movement Science. Abingdon, U.K.: Routledge, 2003.

[30] V. Zatsiorsky, "Biomechanical characteristics of the human body," in Biomechanics and Performance in Sport. Schorndorf, Germany: Karl Hofmann, 1983, pp. 71-83.

[31] V. Zatsiorsky and V. Seluyanov, "The mass and inertia characteristics of the main segments of the human body," in Biomechanics VIII-B. Champaign, IL, USA: Human Kinetics, 1983, pp. 1152-1159.

[32] V. Zatsiorsky and V. Seluyanov, "Estimation of the mass and inertia characteristics of the human body by means of the best predictive regression equations," in Biomechanics IX-B. Champaign, IL, USA: Human Kinetics, 1985, pp. 233-239.

[33] T. Ackland, P. Henson, and D. Bailey, "The uniform density assumption: Its effect upon the estimation of body segment inertial parameters," Int. J. Sports Biomech., vol. 4, pp. 146-155, 1988.

[34] H. K. Huang and F. R. Suarez, "Evaluation of cross-sectional geometry and mass density distributions of humans and laboratory animals using computerized tomography," J. Biomech., vol. 16, pp. 821-832, 1983.

[35] C. K. Cheng, "Segment inertial properties of Chinese adults determined from magnetic resonance imaging," Clinical Biomech., vol. 15, pp. 559$566,2000$.

[36] P. E. Martin, M. Mungiole, M. W. Marzke, and J. M. Longhill, "The use of magnetic resonance imaging for measuring segment inertial properties," J. Biomech., vol. 22, pp. 367-376, 1989.

[37] M. Mungiole and P. E. Martin, "Estimating segment inertial properties: Comparison of magnetic resonance imaging with existing methods," $J$. Biomech., vol. 23, pp. 1039-1046, 1990.

[38] M. Rossi, A. Lyttle, and B. Blanksby, "Body segment inertial parameters of elite swimmers using DXA and indirect methods," J. Sport Sci. Med., vol. 12, no. 4, pp. 761-775, 2013.

[39] R. Dumas, L. Cheze, and J. P. Verriest, "Adjustments to McConville et al. and Young, et al. body segment inertial parameters," J. Biomech. vol. 40, no. 3, pp. 543-553, 2007.

[40] R. Dumas, L. Cheze, and J. P. Verriest, "Corrigendum to 'Adjustments to McConville et al. and Young et al. body segment inertial parameters' [J. Biomech. 40 (2007) 543-553]", J. Biomech., vol. 40, no. 7, pp. 1651-1652, 2007.
[41] J. T. McConville and C. E. Clauser, "Anthropometric assessment of the mass distribution characteristics of the living human body," in Proc. 6th Congr. Int. Ergonom. Assoc. Annu. Meeting, 1976, pp. 379-383.

[42] J. T. McConville, T. D. Churchill, I. Kaleps, C. E. Clauser, and J. Cuzzi, "Anthropometric relationships of body and body segment moments of inertia," Aerosp. Med. Res. Lab., Wright-Patterson Air Force Base, OH, USA, WADC Tech. Rep. AFAMRL-TR-80-119, 1990.

[43] J. W. Young, R. F. Chandler, C. C. Snow, K. M. Robinette, G. F. Zehner, and M. S. Lofberg, "Anthropometric and mass distribution characteristics of the adults female," FAA Civil Aeromed. Inst., Oklahoma City, OK, USA, Tech. Rep. FA-AM-83-16, 1983.

[44] Human Body Properties Database. [Online]. Available: https://www. dh.aist.go.jp/database/properties/index-e.html

[45] H. W. Stoudt, "The anthropometry of the elderly," Human Factors, vol. 23, no. 1, pp. 29-37, 1981.

[46] K. Kaneko, F. Kanehiro, S. Kajita, H. Hirukawa, T. Kawasaki, M. Hirata, K. Akachi, and T. Isozumi, "Humanoid robot HRP-2," presented at the IEEE Int. Conf. Robot. Autom., New Orleans, LA, USA, 2004.

[47] Cortex 2.1 Reference Manual. [Online]. Available: http://www. motionanalysis.com/html/industrial/cortex.html

[48] S. A. Skogstad, K. Nymoen, M. Hovin, S. Holm, and A. R. Jensenius, "Filtering motion capture data for real-time applications," in Proc. Int Conf. New Interfaces Musical Expression, Daejeon, South Korea, 2013 pp. 196-197.

[49] D. G. E. Robertson, G. E. Caldwell, J. Hamill, G. Kamen, and S. N. Whittlessey, Research Methods in Biomechanics, 2nd ed. Champaign, IL, USA: Human Kinetics, 2014.

[50] M. Rynkiewicz, T. Rynkiewicz, P. Zurek, E. Ziemann, and R. Szymanik, "Asymmetry of muscle mass distribution in tennis players," TRENDS Sport Sci., vol. 1, no. 20, pp. 47-53, 2013.

[51] C. English, H. McLennan, K. Thoirs, A. Coates, and J. Bernhardt, "Loss of skeletal muscle mass after stroke: a systematic review," Int. J. Stroke., vol 5, no. 5, pp. 395-402, 2010.

[52] K. Ayusawa, G. Venture, and Y. Nakamura, "Real-time implementation of physically consistent identification of human body segments," in Proc. IEEE Int. Conf. Robot. Autom., 2011, pp. 6282-6287. 